\title{
Propiedades físicas de nanoestructuras de GaSb para aplicaciones en espintrónica
}

\author{
NoRma Diana SARMiento CRUZ ${ }^{1}$ \\ ISMAEL FERNANDO RODRÍGUEZ BALLESTEROS ${ }^{1}$ \\ HEIDDY PAOLA Quiroz GaITÁN ${ }^{1}$ \\ ANDERSON DuSSÁN CUENCA ${ }^{1}$ \\ Ximena Audrey Velásquez MoyA ${ }^{1}$
}

\section{Resumen}

En este trabajo se fabricaron películas delgadas nanoestructuradas de GaSb por el método de pulverización catódica asistidas por campo magnético sobre sustratos de vidrio e ITO. Se realizaron procesos de recocido posterior a la preparación y bajo condiciones de alto vacío que evitaran la incorporación de átomos de oxígeno presentes en la atmósfera. A partir de medidas de difracción de rayos X se pudo establecer una estructura tipo blenda de Zinc y fases de InO asociadas al sustrato ITO. Los procesos de recocido permitieron evidenciar una mejora significativa en la cristalinidad del material siendo éste menos amorfo cuando la temperatura de recocido ( $\mathrm{Tr}$ ) fue de $673 \mathrm{~K}$. Un valor de la brecha de energía prohibida variando entre 0.75 y 0.85 eV fue obtenido en muestras de GaSb cuando la Tr cambió entre $300 \mathrm{~K}$ y 673 $\mathrm{K}$, respectivamente. Medidas de microscopia electrónica de barrido y fuerza atómica permitieron obtener información de la morfología en la superficie del material.

Palabras claves: Pulverización catódica, blenda de Zinc, nanoestructuras, espintrónicos, películas delgadas.

1 Universidad Nacional de Colombia, sede Bogotá, Colombia.

Autor de correspondencia: Quiroz Gaitán, H.P. (Heiddy Paola): Universidad Nacional de Colombia, Facultad de Ciencias, Departamento de Física, Grupo de Materiales Nanoestructurados y sus Aplicaciones: Cra $30 \mathrm{~N}^{\circ} 45-03$, Edificio 404, Lab. 121C. Ciudad Universitaria, Bogotá D.C., 11001 - Colombia Teléfono: +057-1-3165000 Ext. 13088. Correo electrónico: hpquirozg@unal.edu.co
Historia del artículo:

Artículo recibido: 20-XI-2018 / Aprobado: 12-XII-2018

Disponible online: 3 de febrero de 2019

Discusión abierta hasta octubre de 2020 


\section{Physical Properties of GaSb Nanostructures for Spintronic Applications}

\section{Abstract}

In this work, GaSb thin films were prepared by DC magnetron sputtering method using Soda Lime-type glass and ITO (Indium tin oxide), as substrates. Annealing processes were carried out under high vacuum conditions to avoid the incorporation of oxygen (O) atoms present in the atmosphere. From X-ray diffraction (XRD) measurements it was possible to establish a Zinc-blende type structure and InO phases associated to the ITO substrate. The annealing processes showed a significant improvement in the crystallinity of the material being less amorphous when the annealing temperature (Tr) was $673 \mathrm{~K}$. A value of the gap energy ranging from 0.75 to $0.85 \mathrm{eV}$ was obtained in samples of GaSb when the Tr changed between $300 \mathrm{~K}$ and $673 \mathrm{~K}$, respectively. Scanning Electron Microscopy (SEM) and atomic force microscopy (AFM) measurements provided information on the surface morphology of the material.

Keywords: Sputtering, zinc blende, nanostructurs, spintronics, thin films.

\section{Propriedades físicas de nanoestruturas GaSb para aplicações em spintrônica}

\section{Resumo}

Neste trabalho, os filmes finos de GaSb foram preparados pelo método de pulverização catódica assistido por campo magnético em substratos de vidro e ITO (óxido de estanho de índio). Após os processos de recozimento foram realizados em condições de alto vácuo que evitam a incorporação de átomos de oxigênio (0) presentes na atmosfera. A partir de medições de difração de raios X (XRD), foi possível estabelecer uma estrutura semelhante ao Zenz Blende e as fases InO associadas ao substrato ITO. Os processos de recozimento mostraram uma melhoria significativa na cristalinidade do material sendo menos amorfa quando a temperatura de recozimento ( $\mathrm{Tr}$ ) foi de $673 \mathrm{~K}$. Foi obtido um valor do intervalo de energia proibido variando de 0,75 a 0,85 eV em amostras de GaSb quando o Tr mudou entre $300 \mathrm{~K}$ e $673 \mathrm{~K}$, respectivamente. As medições de microscopia eletrônica de varredura (SEM) e microscopia de força atômica (AFM) forneceram informações sobre a morfologia da superfície do material.

Palavras-chaves: Sputtering, Blende zinco, nanoestruturas, spintrónica, filmes finos.

\section{Introducción}

En el desarrollo de nuevos materiales, el estudio de las propiedades físicas de semiconductores III-V tales como GaAs, InAs y InSb (Mosher y Soukup, 1982; Hongwei et al., 1998; Carroll y Spivak, 1966), ha sido de gran importancia para el desarrollo de aplicaciones en dispositivos ópticos (Bonilla-Marin,
2007), electro-ópticos (Kluth et al., 2005; Glemza et al., 2017) celdas solares (Benyettou et al., 2015), entre otros. En particular, el semiconductor GaSb se ha utilizado en la fabricación de detectores de luz láser, dispositivos de alta frecuencia, sensores de radiación infrarroja y celdas solares, debido a que responde a un amplio rango de longitudes de onda en la región del infrarrojo (Benyettou et al., 2015). 
Sin embargo, desde 1996 (Ohno et al., 1996) se ha dado mayor preponderancia al compuesto $\mathrm{GaSb}$ considerándolo como una matriz semiconductora con propiedades interesantes para la obtención de semiconductores magnéticos diluidos (Diluted Magnetic Semiconductors, DMS por sus siglas en inglés) y su aplicación en el desarrollo de la espintrónica (Adhikari y Basu, 1996). Aunque los reportes en la fabricación de estos materiales se basan en técnicas como el crecimiento epitaxial por haces moleculares (MBE Molecular beam epitaxial por sus siglas en inglés) (Papaj et al., 2014) o depósito por láser pulsado (PLD Pulsed Laser Deposition, por sus siglas en inglés) (Rout et al., 2013), en los últimos años se han estudiado otros métodos de depósito como la pulverización catódica (sputtering, témino en inglés asociado a este tipo de depósito) (Calderón, Quiroz y Dussan, 2017) o químicos como CVD (Chemical Vapor Deposition, por sus siglas en inglés) (Pärna et al., 2011), que al incluirle átomos de elementos de transición como el Manganeso (Mn) y el Niquel (Ni) presentan comportamientos ferromagnéticos y ferrimagnéticos asociados a la formación de fases binarias entre el elemento de transición y los elementos del sustrato (Calderón, Mesa y Dussan, 2017).

En este trabajo se presenta un estudio de las propiedades estructurales, ópticas y morfológicas de películas delgadas de GaSb fabricadas a partir del método de pulverización catódica asistida por campo magnético en corriente continua o comúnmente conocido como "DC Magnetron Sputtering". Las propiedades estructurales, partiendo por la identificación de fases cristalinas, los parámetros de red y el tamaño del cristalito fueron estudiados a partir de medidas de XRD, antes y después de la realización de procesos de recocido insitu. Adicionalmente, se presenta un estudio, a partir de medidas de SEM y AFM, del cambio en la morfología de la superficie del material al someter las muestras a ataques electroquímicos. Las constantes ópticas (índice de refracción, coeficiente de absorción, energía de brecha prohibida, espesor, etc) fueron obtenidas a partir de medidas de transmitancia espectral. Una correlación entre las propiedades estudiadas y los parámetros de síntesis es presentada.

\section{Materiales y métodos}

Películas delgadas de GaSb fueron fabricadas utilizando el método de DC magnetrón Sputtering, usando un blanco Ga(36.5 Wt\%)Sb(63.5 Wt\%) con una pureza del 99.99\%. Las muestras fueron depositadas sobre sustratos de ITO (Indium tin oxide) y vidrio tipo Boro-silicato, éstos previamente sometidos a un proceso de limpieza con Alconox. Se sometieron a procesos de recocido entre $523 \mathrm{~K}$ y $623 \mathrm{~K}$ in situ durante $2 \mathrm{~h}$, en condiciones de alto vacío ( $\left.\sim 10^{-6} \mathrm{Torr}\right)$, posterior a la etapa de preparación, usando como fuente de calefacción lámparas IR y un PID para el control de la temperatura.

En el proceso de síntesis de las muestras, la potencia aplicada al blanco de GaSb fue de $100 \mathrm{~W}$, la presión de trabajo en la cámara fue $2.5 \times 10^{-2}$ Torr con una distancia sustrato-blanco de $7 \mathrm{~cm} \mathrm{y}$ un tiempo de depósito de 5 y 15 min.

Las muestras fueron caracterizadas a través de medidas de difracción de rayos $\mathrm{X}$ en una configuración Bragg-Brentano en el rango de $10^{\circ} \leq 2 \theta \leq 90^{\circ}$ utilizando un difractómetro de Rayos X Policristal $X$ 'Pert Pro de PANalytical, equipado con una fuente de $\mathrm{Cu}-\mathrm{K} \alpha$ : $1,540598 \AA$, a una diferencia de potencial de $40 \mathrm{kV}$, corriente de $40 \mathrm{~mA}$ y un detector $\mathrm{X}^{\prime}$ Celerator. El software empleado para la identificación de fases presentes en las muestras fue el X'Pert HighScore Plus usando refinamiento Rietveld.

Para la obtención de las propiedades ópticas se utilizó un espectrofotómetro marca Cary 5000 UV-VIS-NIR de alto rendimiento en el rango desde $300 \mathrm{~nm}$ a $2500 \mathrm{~nm}$, trabajando a presión atmosférica y temperatura ambiente. Para estudiar la morfología de las películas se utilizó un microscopio electrónico de barrido VEGA3 SB con una fuente termoiónica de tungsteno, un voltaje de aceleración de $5 \mathrm{kV}$, en condiciones de alto vacío ( $\sim 10^{-6}$ mbar). Fueron complementadas con un estudio de la topografía a partir de un microscopio 
de fuerza atómica Asylum Research MFP 3D Bio en modo "tapping" o de contacto intermitente.

\section{Resultados y discusión}

En la Figura 1a se presentan los patrones de DRX de las muestras sin recocer y recocidas a $573 \mathrm{~K}$ y $623 \mathrm{~K}$, depositadas sobre vidrio e ITO. Se puede observar que la cristalización de las películas depende fuertemente del sustrato utilizado y se evidencia en un aumento de la cristalinidad en la muestra depositada sobre ITO, favorecidas por las fases $\mathrm{SnO}_{2}$ y InO. En la Figura $1 \mathbf{b}$ se presenta la estructura cristalina del compuesto GaSb, el cual tiene una estructura cristalina cúbica con grupo espacial F-43m.

Adicionalmente, es posible observar la contribución amorfa en el patrón de DRX en todas las muestras, reducida sustancialmente por la elevación de la temperatura en los procesos de recocido y las propiedades cristalinas del sustrato, las cuales favorecen la nucleación de fases de GaSb y el crecimiento de películas policristalinas.

En la Figura 2 se presentan las medidas de transmitancia espectral y reflectancia en función de la longitud de onda de películas de GaSb variando la temperatura de recocido entre ambiente y $623 \mathrm{~K}$.

Figura 1. a) Patrón de DRX de las películas de GaSb variando la temperatura de recocido sobre sustratos de vidrio e ITO. b) Estructura blenda de Zinc de la fase GaSb con parámetro de red $a=6.09 \AA \AA$.

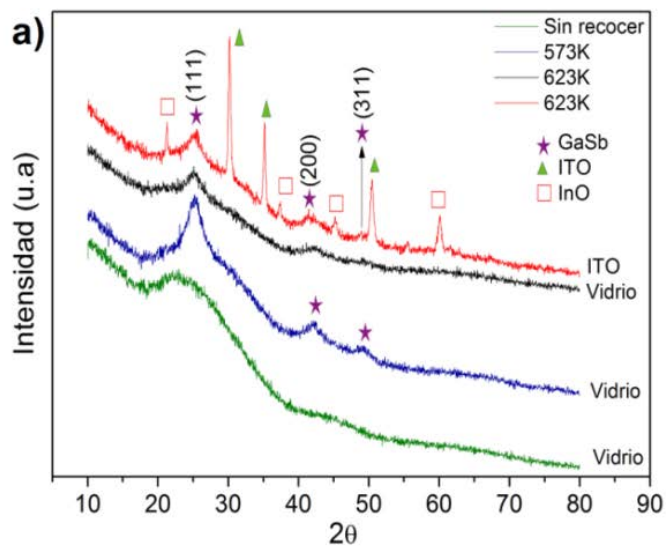

b)

$a=b=c=6,09 \AA$

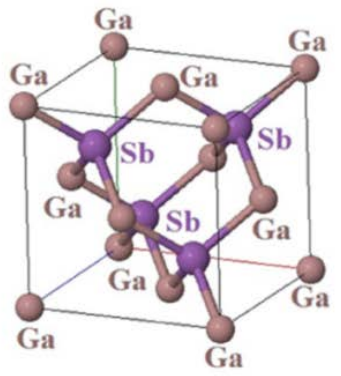

Figura 2. Medidas de a) transmitancia espectral y b) reflectancia en función de la longitud de onda para las muestras de GaSb variando la temperatura de recocido, $T_{r}$.
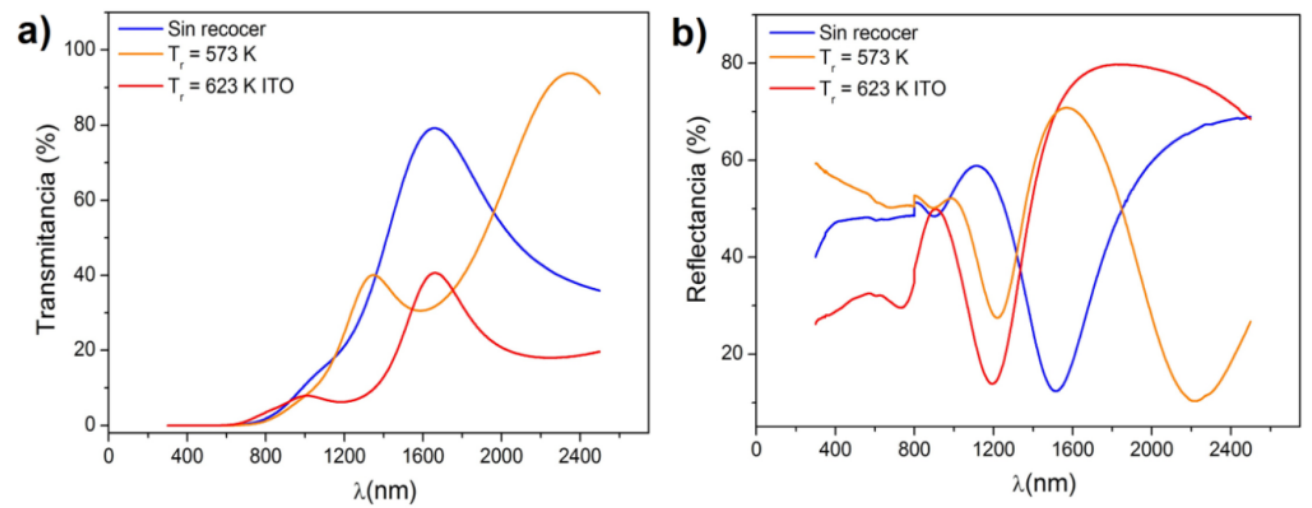
Se puede observar, a partir de la Figura 2 la presencia de franjas de interferencia asociada a los procesos de interferencia entre la película y el sustrato, que se ven afectadas por el espesor del material y los procesos de absorción de la luz en el semiconductor.

A partir de las medidas de transmitancia y reflectancia fue posible determinar el coeficiente de absorción $\alpha$ de las películas utilizando la ley de Beer (Quiroz, 2014), y por lo tanto el "gap" (Eg). En la Figura 3 se presentan las curvas de absorción para las películas de GaSb sin recocido y con recocido de $573 \mathrm{~K}$.

De acuerdo a la Figura 3, los valores "band gap" son reportados en la Tabla 1 donde se puede observar un corrimiento hacia valores mayores de $0.72 \mathrm{eV}$ cuando aumenta la temperatura de recocido, con una diferencia porcentual de $4.2 \%$ con respecto al valor reportado para el "band gap" del GaSb (Calderón, Mesa y Dussan, 2017). Sin embargo, al aumentar el tiempo de depósito se observa una disminución del "gap" debido al aumento del espesor de las películas, lo que favoreció la cristalización de las películas.

En la Figura 4 se presenta el índice de refracción (n) en función de la longitud de onda para las muestras con variación de recocido, obtenido a partir de las medidas de transmitancia espectral. Se puede observar un aumento de $\mathrm{n}$ cuando a las muestras se les realizan recocidos, debido a que aumenta la dispersión al incrementarse la longitud de onda de acuerdo a que la región de absorción de las películas de GaSb se encuentra en el infrarrojo, por lo que a longitudes de onda mayores a $850 \mathrm{~nm}$, el índice de refracción es casi constante.

Figura 3. Curvas de absorción para las películas de GaSb. a) Sin recocido y b) $\mathrm{T}_{\mathrm{r}}=573 \mathrm{~K}$ de recocido. El inset evidencia la región donde se observa el gap de la película sin recocido
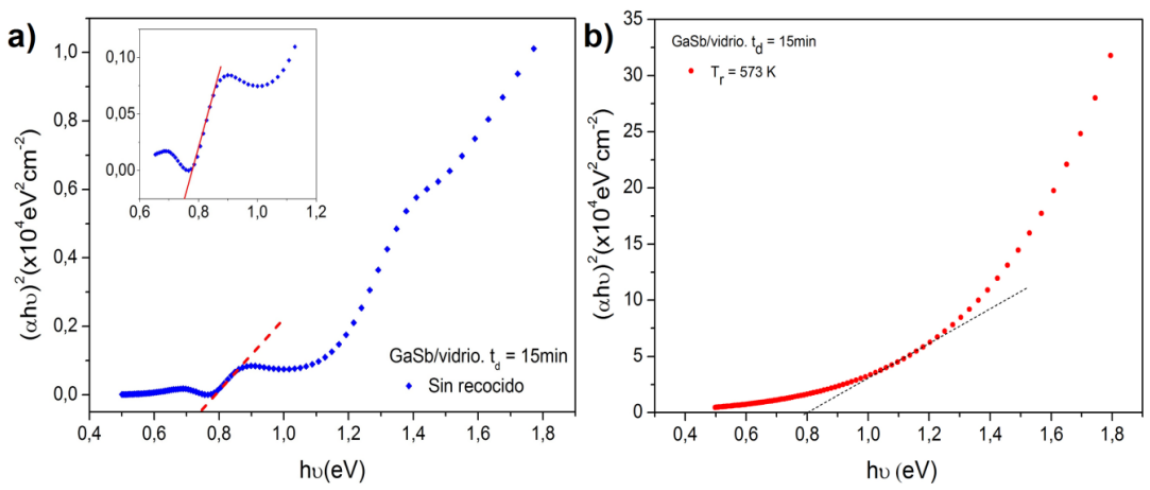

TABLA 1. VALORES DE LA BRECHA DE ENERGÍA PROHIBIDA O “BAND GAP" OBTENIDOS PARA LAS PELÍCULAS DE GaSb VARIANDO T

\begin{tabular}{|c|c|c|c|}
\hline Muestra & $E_{g} \pm 0.01 e V$ & $\mathrm{n}(\lambda=1000 \mathrm{~nm})$ & $a\left(\mathrm{~cm}^{-1}\right)$ \\
\hline Sin recocer & 0.75 & 5.16 & $3.87 \times 10^{6}$ \\
\hline $573 K$ & 0.80 & 4.89 & $3.29 \times 10^{6}$ \\
\hline $623 K$ & 0.85 & 7.80 & $4.45 \times 10^{6}$ \\
\hline \multicolumn{4}{|c|}{$t_{d}$} \\
\hline Muestra & $\mathrm{Eg} \pm 0.01 \mathrm{eV}$ & $\mathrm{n}(\lambda=1000 \mathrm{~nm})$ & $a\left(\mathrm{~cm}^{-1}\right)$ \\
\hline $5 \mathrm{~min}$ & 1.25 & 3.12 & $9.77 \times 10^{6}$ \\
\hline $15 \mathrm{~min}$ & 0.85 & 4.89 & \\
\hline
\end{tabular}


Figura 4. Índice de refracción en función de la longitud de onda para las películas delgadas de GaSb sobre vidrio variando $\mathrm{T}_{\mathrm{r}}$ entre $300 \mathrm{~K}$ y $623 \mathrm{~K}$.

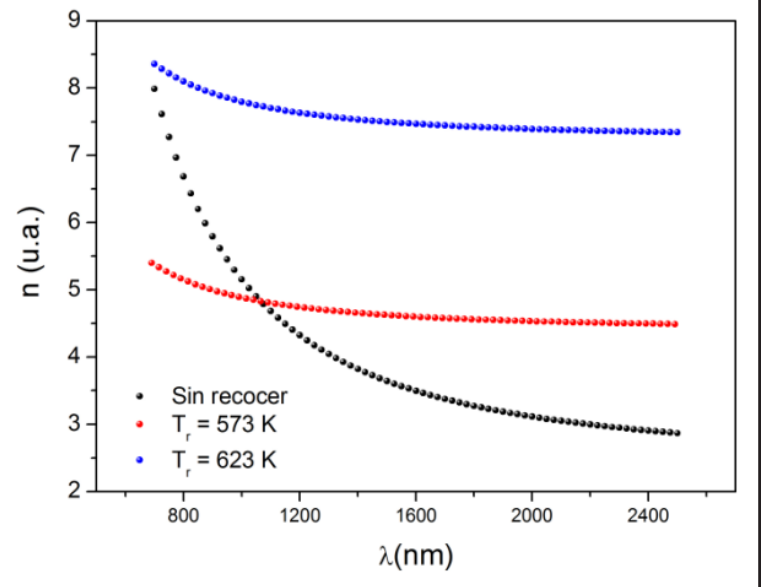

Por otro lado, se realizaron medidas de microscopia electrónica de barrido para estudiar la superficie de las películas. En la Figura $\mathbf{5}$ se presentan las micrografías de las películas en las que se variaron las temperaturas de recocido entre $623 \mathrm{~K}$ y $648 \mathrm{~K}$.

Se puede observar que la superficie de las películas de GaSb son muy homogéneas y no se ven considerablemente afectadas por las variaciones de temperatura. Esto es debido a que el método de depósito (DC magnetrón sputtering) favorece la homogeneidad del depósito del material sobre los sustratos y la formación de granos o islas se ven gobernadas por el tiempo de depósito.

En la Figura 6 se presenta la micrografía SEM y AFM de la muestra con un tiempo de 15 min y un recocido de $623 \mathrm{~K}$, donde se observa el espesor de la película y la topografía. Es posible identificar la formación de pequeños clústeres en la superficie, asociado al estado de nucleación que ocurre durante el depósito, y que pueden afectar las medidas de transmitancia y reflectancia de las películas (Figura 2 y 4 ).

Debido a que las especies involucradas en el depósito $(\mathrm{Ga}, \mathrm{Sb})$ no realizan una difusión hacia el sustrato, se puede evidenciar estos procesos de nucleación como una disminución de la movilidad del Ga y Sb generando formaciones de pequeños clústeres distribuidos homogéneamente sobre toda la superficie y de escala nanométrica (Wasa, Kitabatake y Adachi, 2004) y que se ven afectados también por el espesor de la película delgada. El espesor de las películas fue medido a partir de las micrografías SEM y variaron entre $180 \mathrm{~nm}$ y $280 \mathrm{~nm}$ para las muestras con tiempo de depósito de 5 min y 15 min, respectivamente (ver Tabla 2 ).

Figura 5. Micrografía SEM de la superficie de la muestra de GaSb con $T_{r}$ a) $623 \mathrm{~K}$ y b) $648 \mathrm{~K}$, con un tiempo de depósito de $15 \mathrm{~min}$.

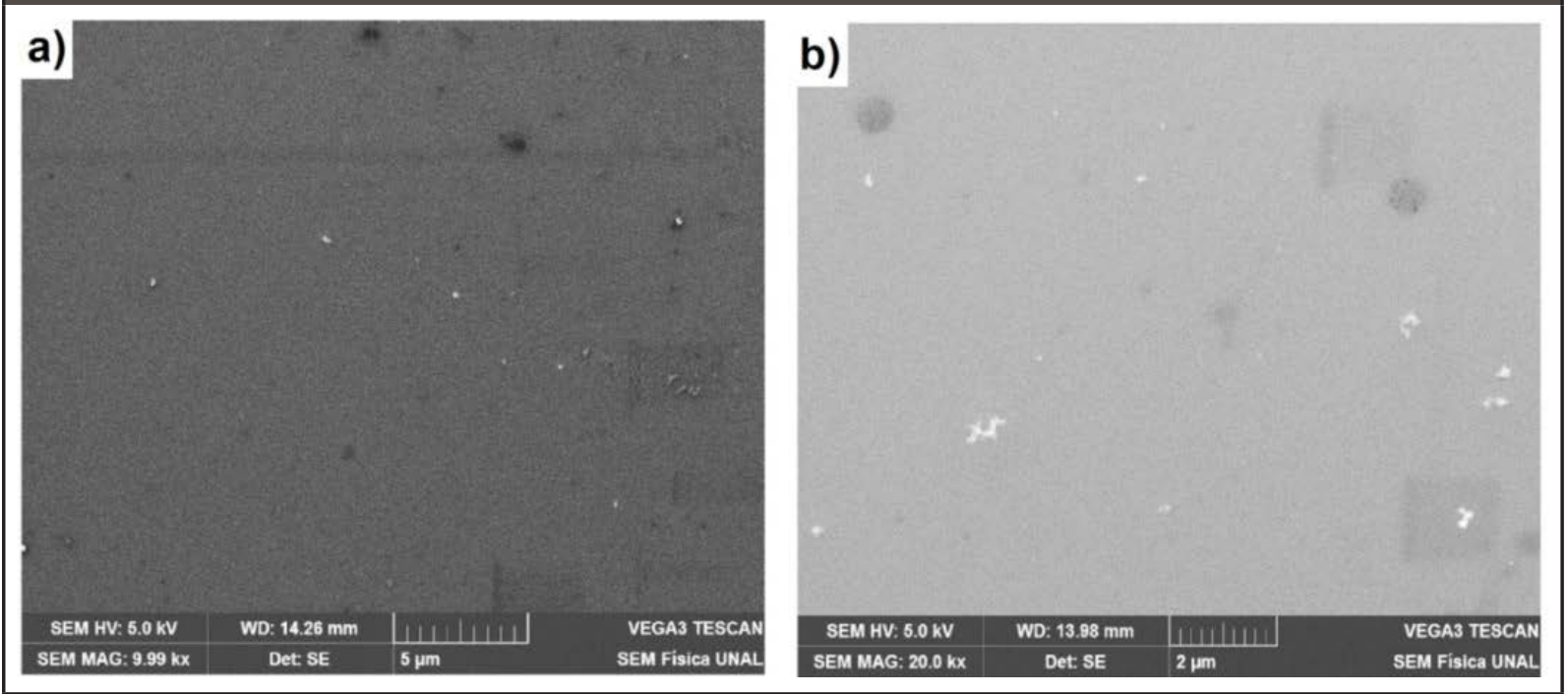


Figura 6. Micrografía SEM y AFM de la muestra depositada con $\mathrm{td}=15 \mathrm{~min}$. En la parte superior se observa la micrografía SEM de la superficie de la película y su reconstrucción en 3D.

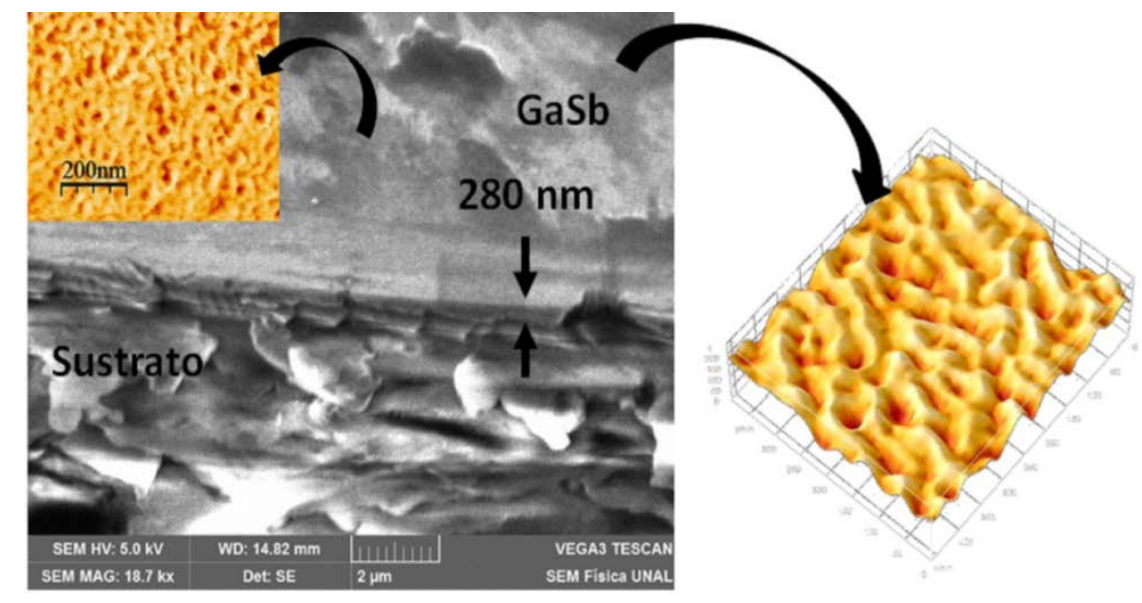

Figura 7. Micrografía SEM y AFM de la película de GaSb con un $\mathrm{Td}=5$ min y $\operatorname{Tr}=623 \mathrm{~K}$ después del anodizado electroquímico. Se remarca la región que fue atacada y la formación de poros en la superficie.

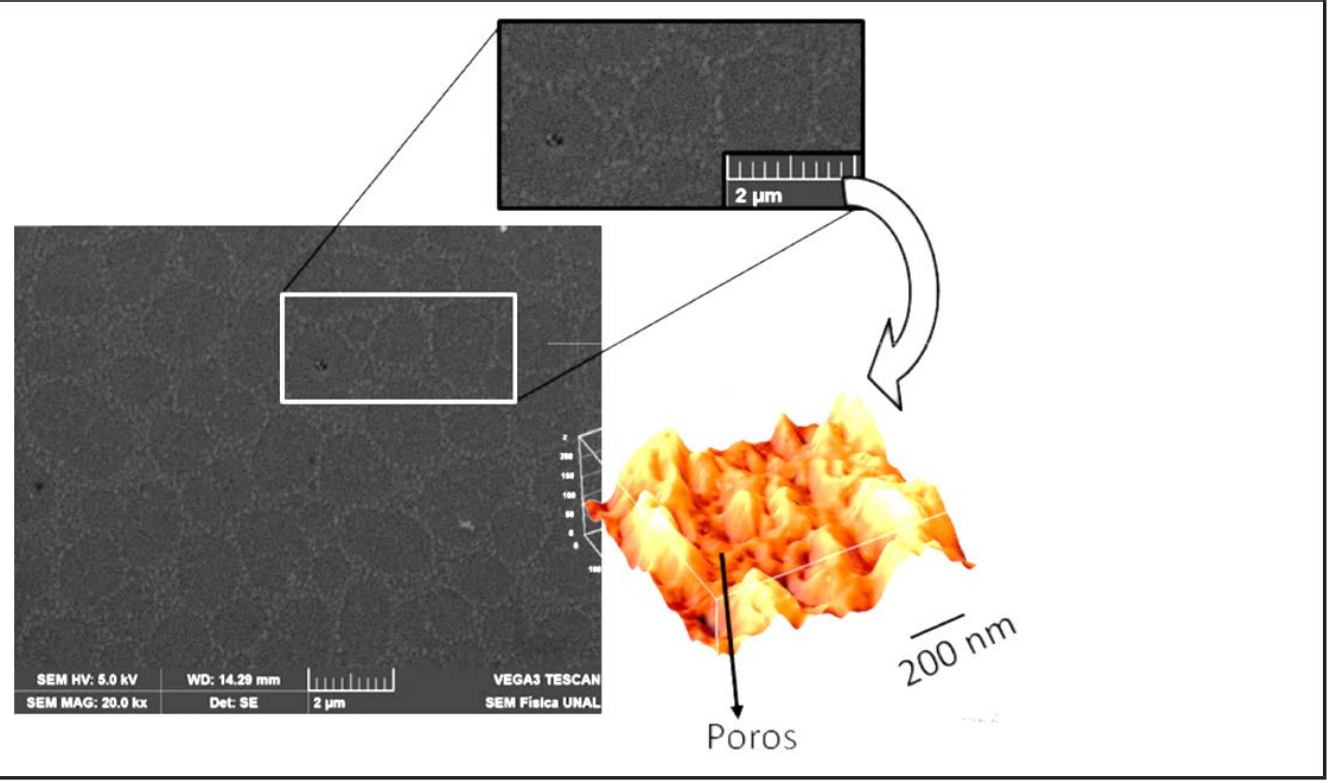

Por lo tanto, las nanoestructuras que gobiernan la superficie de las películas de GaSb pueden modificarse con los parámetros de síntesis y el método de depósito (Bonilla-Marin, 2007). Sin embargo, existen otras formas de modificar la superficie, entre la cuales se encuentran ataques por métodos químicos. En la Figura 7 se presenta la micrografía SEM y AFM de la película de GaSb con un tiempo de depósito de 15 min y recocido de $623 \mathrm{~K}$ después de realizar anodizado electroquímico.
Se puede observar la formación de poros en la superficie después del ataque realizado por medio de anodizado electroquímico utilizando ácido clorhídrico ( $\mathrm{HCl}$ ) en concentración del $33,9 \%$ en $10 \mathrm{ml}$ ( $9 \mathrm{ml}$ de $\mathrm{HCl}$ y $1 \mathrm{ml}$ de agua desionizada). Como cátodo se utiliza un alambre de platino $(\mathrm{Pt})$ y se suministró una corriente de $1 \mathrm{~mA}$. A partir de las medidas de microscopia de fuerza atómica fue posible determinar la rugosidad de las muestras después del anodizado (ver Tabla 2). 
TABLA 2. TIEMPO DE DEPÓSITO, TEMPERATURA DE RECOCIDO, CORRIENTE APLICADA Y RUGOSIDAD DE LAS PELÍCULAS DE GaSb.

\begin{tabular}{c|c|c|c|c|c|} 
Muestra & $\begin{array}{c}\text { Tiempo de depósito } \\
\text { (min) }\end{array}$ & $\begin{array}{c}\text { Temperatura de } \\
\text { recosido (K) }\end{array}$ & $\begin{array}{c}\text { Corriente } \\
\text { aplicada (mA) }\end{array}$ & $\begin{array}{c}\text { Rugosidad } \\
\text { RMS (nm) }\end{array}$ & Espesor (nm) \\
\hline 1 & 15 & 623 & 0.5 & 3,02 & 280 \\
\hline 2 & 15 & 648 & 1 & 7,38 & 280 \\
\hline 3 & 15 & 648 & 0.15 & 1,043 & 280 \\
\hline
\end{tabular}

\section{Conclusiones}

Se fabricaron películas delgadas de GaSb por el método de DC magnetrón sputtering, variando el tiempo de depósito y temperaturas de recocido in situ. Se estudiaron los cambios en las propiedades estructurales antes y después de los recocidos y utilizando diferentes sustratos (vidrio e ITO). Se correlacionaron los parámetros de síntesis con el "gap", el índice de refracción y las medidas ópticas. Se realizaron modificaciones de la topografía de las películas a partir de anodizado electroquímico, evidenciando la formación de nanoestructuras porosas en la superficie y que pueden afectar las propiedades ópticas de las películas de GaSb.

\section{Agradecimientos}

Este trabajo fue soportado por la Universidad Nacional de Colombia - COLCIENCIAS con el proyecto Código Quipú № 201010020958.

\section{Referencias}

Adhikari, T.; Basu, S. (1996). Carrier-induced ferromagnetic ordering in $\mathrm{Ga}_{1-\mathrm{x}} \mathrm{Mn}_{\mathrm{x}} \mathrm{Sb}$, a new III-Mn-V semimagnetic semiconductor. Journal of Magnetism and Magnetic Materials, 161, pp. 282-286. [Online] Disponible en: https://doi.org/10.1016/S03048853(95)01281-8 [Consultado 26 de septiembre de 2017].

Benyettou, F.; Aissat, A.; Benamar, M.A.; Vilcot, J.P. (2015). Modeling and Simulation of GaSb/GaAs Quantum Dot for Solar Cell. Energy Procedia, 74, pp. 139-147. [Online] Disponible en: https://doi.org/10.1016/j. egypro.2015.07.535 [Consultado 26 de septiembre de 2017].
Bonilla-Marin, Jose Lauro. (2007). Obtención y caracterización del semiconductor poroso antimonio de galio con impurezas de telurio, tesis (Maestro en Tecnología Avanzada), Santiago de Queretaro, Instituto Politécnico Nacional, 71 pp. Disponible en: http://tesis.ipn.mx/bitstream/ handle/123456789/1093/2321_2007_CICATAQUERETARO_MAESTRIA_bonilla_marin_joselauro. pdf?sequence=1\&isAllowed=y [Consultado 26 de septiembre de 2017].

Calderón, Jorge A.; Mesa, F; Dussan A. (2017). Magnetoelectric and transport properties of (GaMn)Sb thin films: A ferrimagnetic phase in dilute alloys. Applied Surface Science, 396, pp. 1113-1118. [Online] Disponible en: https://doi.org/10.1016/j.apsusc.2016.11.096 [Consultado 26 de septiembre de 2017].

Calderón, Jorge A.; Quiroz, Heiddy P.; Dussan, A. (2017). Optical and structural properties of GaSb doped Mn based diluted magnetic semiconductor thin films grown via DC magnetron sputtering. Advanced Materials Letters, 8(5), pp. 650-655 [Online] Disponible en: 10.5185/amlett.2017.7110 [Consultado 26 de septiembre de 2017].

Carroll, J.A.; Spivak, J.F. (1966). Preparation of high mobility InSb thin films. Solid-State Electronics, 9(5), pp. 383-384. [Online] Disponible en: 10.1016/00381101(66)90152-3 [Consultado 26 de septiembre de 2017].

Glemza, Justinas; Pralgauskaite, Sandra; Palenskis, Vilius; Matukas, Jonas. (2017). Low frequency noise investigation of 2-3 $\mu \mathrm{m}$ GaSb-based laser diodes. Solid-State Electronics, 133, pp. 70-77. [Online] Disponible en: https://doi.org/10.1016/j. sse.2017.05.002 [Consultado 26 de septiembre de 2017]. 
Hongwei, Z.; Yiping, Z.; Jianrong, D.; Zhanping, Z.; Liang, P.; Meiying, K. (1998). Growth and transport properties of InAs thin films on GaAs. Journal of Crystal Growth, 191(3). pp. 361-364. [Online] Disponible en: https://doi.org/10.1016/S00220248(98)00161-4 [Consultado 26 de septiembre de 2017].

Kluth, S. M.; Jojannessen, B.; Kluth, P.; Glover, C. J.; Foran, G. J.; Ridway, M. C. (2005). EXAFS comparison of crystalline/continuous and amorphous/porous GaSb. Nuclear Instruments and Methods in Physics Research Section B: Beam Interactions with Materials and Atoms, 238(1-4), pp. 264-267. [Online] Disponible en: https://doi.org/10.1016/j. nimb.2005.06.060 [Consultado 26 de septiembre de 2017].

Mosher, D.M.; Soukup, R.J. (1982). The fabrication of both n-type and p-type GaAs thin films deposited by troide sputtering. Thin films, 98(3), pp. 215-228. [Online] Disponible en: https://doi. org/10.1016/0040-6090(82)90404-7 [Consultado 26 de septiembre de 2017].

Ohno, H.; Shen, A.; Matsukura, F.; Oiwa, A.; Endo, A.; Katsumoto, S.; Iye, Y. (1996). (Ga,Mn)As: A new diluted magnetic semiconductor based on GaAs. Applied Physics Letters, 69(3), pp. 363-365. [Online] Disponible en: http://dx.doi.org/10.1063/1.118061 [Consultado 26 de septiembre de 2017].

Papaj, M.; Kobak, J.; Rousset, J.G.; Janik, E.; Nawrocki, M.; Kossacki, P.; Golnik, A.; Pacuski, W. (2014). Photoluminescence studies of giant Zeeman effect in MBEgrown cobalt-based dilute magnetic semiconductors. Journal of Crystal Growth, 401, pp. 644-647. [Online] Disponible en: https://doi.org/10.1016/j. jcrysgro.2014.01.077 [Consultado 26 de septiembre de 2017].

Pärna, R.; Joost, U.; Nõmmiste, E.; Käämbre, T.; Kikas, A.; Kuusik, I.; Hirsimäki, M.; Kink, I.; Kisand, V. (2011). Effect of cobalt doping and annealing on properties of titania thin films prepared by sol-gel process. Applied Surface Science, 257, pp. 6897-6907. [Online] Disponible en: https://doi.org/10.1016/j. apsusc.2011.03.026 [Consultado 26 de septiembre de 2017].

Quiroz, Heiddy P. (2014). Preparación y Estudio de las Propiedades Estructurales, Ópticas y Morfológicas de Nanotubos de $\mathrm{TiO}_{2}$ para su Aplicación en Sen- sores Ópticos Tesis (Maestría en Ciencias - Física) Bogotá, Universidad Nacional de Colombia, 103 pp. Disponible en: http://www.bdigital.unal.edu. co/47304/1/1072655319.2014.pdf [Consultado 26 de septiembre de 2017].

Rout, S.; Popovici, N.; Daluia, S.; Paramês, M.L.; da Silva, R.C.; Silvestre, A.J.; Conde, O. (2013). Phase growth control in low temperature PLD Co: $\mathrm{TiO}_{2}$ films by pressure. Current Applied Physics, 13(4), pp. 670-676. [Online] Disponible en: https://doi. org/10.1016/j.cap.2012.11.005 [Consultado 26 de septiembre de 2017].

Wasa, K.; Kitabatake, M.; Adachi, H. (2004). Thin Film Materials Technology Sputtering of Compound Materials, Springer, pp. 33.

\section{PARA CITAR ESTE ARTÍCULO TO REFERENCE THIS ARTICLE / PARA CITAR ESTE ARTIGO /}

Sarmiento, D.; Rodríguez, I.; Quiroz, H.; Dussán, A. Velásquez, X. (2019). Propiedades físicas de nanoestructuras de GaSb para aplicaciones en espintrónica. Revista EIA, 16(31), enero-junio, pp. 89-97. [Online]. Disponible en: https://doi.org/10.24050/ reia.v16i31.1272 